\title{
Getting down to basics with actin
}

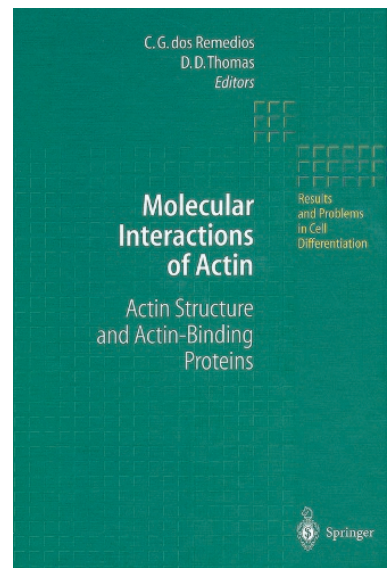

\author{
Molecular Interactions of Actin: Actin Structure and Actin-Binding Proteins \\ edited by C. G. dos Remedios and D. D. Thomas
}

Springer · January 2001

Hardback $£ 82.50 / \$ 120$

$\Lambda$ e ctin is the most abundant protein in eukaryotic cells, at cellular concentraions generally above $100 \mathrm{mM}$. This abundance has made actin a focus of biochemical research since its identification by Straub and Feuer in 1942. A central property of this monomeric protein is that it polymerizes to form helical filaments. In muscle, actin filaments serve as tracks for force generation by myosin motors. Non-muscle cells contain similar force-generating assemblages of actin and myosin, including stress fibres and the contractile ring during cytokinesis. More recently, recognition that actin polymerization itself generates force for a variety of non-muscle cell processes, such as cell motility, phagocytosis, and movement of intracellular pathogens, has raised additional interest in actin's biochemical and cellular properties. Through this research, about 70 families of actinbinding proteins have been identified.

One thing that 50 years of research on actin biochemistry has proved is that the devil is in the details. The polymerization/depolymerization cycle of pure actin in vitro involves multiple steps. In cells, key actin-binding proteins regulate specific steps in this cycle. Other actin-binding proteins crosslink filaments into bundles or networks. The combination of these effects allows for incredible speed and flexibility in controlling actin dynamics, with cells capable of polymerizing or depolymerizing micrometre-thick sheets of densely packed filaments in seconds. Clearly, a detailed understanding of cellular actin functions can only be achieved by understanding how they work at a molecular level.

The aim of Molecular Interactions of Actin is to provide a detailed review of actin's biochemical properties and interactions with other proteins to aid future research. Conceived during the Fourth Pentennial Actin Conference in 1998 on Maui, this book is a compendium of contributions by 15 groups of authors. The first seven chapters discuss aspects of actin structure and in vitro dynamics, providing many often overlooked details rarely found elsewhere. The next six chapters focus primarily on the effects of actin-binding proteins, including $\mathrm{ADF} /$ cofilin and gelsolin, on actin structure and dynamics. The penultimate chapter reviews the growing family of actin-related proteins (Arps), and the last chapter describes signalling pathways that regulate cellular actin-mediated processes. Several of the chapters provide beautifully clear reviews of actin properties in vitro and in cells. Four such chapters are described below.

The chapter by Oosawa on the historical perspective of actin assembly and its interactions is a treat to read for several reasons. First, it provides a first-hand account of the history of actin research from one of the founders of the field. For those seeking the origins of actin biochemical research, this is a great place to start. Second, the chapter supplies valuable references to primary literature measuring basic biochemical/biophysical properties of actin (polymerization in varying buffer conditions, flexibility of filaments, studies of myosin motility). Finally, Oosawa raises a number of unanswered questions concerning changes in actin filaments during myosin-based motility.

The chapter by Belmont and Drubin, on actin structure-function relationships revealed by yeast molecular genetics describes a huge amount of information in an understandable manner. One of the most notable features of this chapter is a four-page table listing every published actin mutant, a description of its biochemical and cellular properties, and references to the primary research articles for each. This comprehensive list allows the authors to examine specific issues in detail in the text (effect of mutations on polymerization dynamics, nucleotide binding and hydrolysis; residues involved in actin-actin interaction; sites of actin-binding protein and drug interactions).

The chapter by Machesky and May on Arps is a good comprehensive review of all
Arp proteins, including Arp1 (in dynactin complex), Arp2 and Arp3 (in Arp2/3 complex), and Arp4 and Arp7/9 ('nuclear' Arps). Because the latest references cited are from 1998, the review deals only briefly with the rapidly developing field of Arp2/3 complex activation by WASp/Scar proteins and other proteins. Even so, the review is unique to my knowledge in that it covers all Arps.

The chapter by Beck, Delley and Hall on the control of the actin cytoskeleton by extracellular signals is an interesting and readable review of signalling pathways from extracellular signal to actin dynamics in three very different eukaryotes: budding yeast, Drosophila and mammals. The juxtaposition of these summaries provides for interesting comparisons. As one reads the chapter, one develops an appreciation for the power of the genetic systems for mapping signalling pathways. Also, brief descriptions of at least 12 possible regulators of actin dynamics downstream of mammalian Rho proteins gives readers the raw materials for deeper investigation.

Three cautionary notes should be provided. First, although this book is part of the series 'Results and Problems in Cell Differentiation', those expecting to find information on the role of actin in differentiation will be disappointed. Second, most chapters require some prior knowledge of the subject, as they hop right into discussion of specific issues. For this reason, the book is more useful for researchers searching for detailed information about the field rather than for undergraduate or even graduate education. Third, results from the past 2 to 3 years are not included in the book, as the most recent references are from 1999 and many chapters only reference through to 1998. This does not detract from most of the chapters, especially if one uses them as starting points for further study. Henry Higgs is in the Salk Institute for Biological Sciences, 10010 North Torrey Pines Road, La Jolla, California 92037, USA e-mail:higgs@perutz.salk.edu 